\title{
Plasma Focusing of High Energy Density Electron and Positron Beams
}

J.S.T. Ng, H.A. Baldis, P. Bolton, P. Chen, D. Cline, W. Craddock, C. Crawford, F.J. Decker, R.C. Field, Y. Fukui, V. Kumar, R. Iverson, F. King, R. Kirby, T. Kotseroglou, K. Nakajima, R. Noble, A. Ogata, P. Raimondi, D. Walz and A.W. Weidemann

This article was submitted to

$9^{\text {th }}$ Advanced Accelerator Concepts Workshop

Santa Fe, NM

June 10-16, 2000

U.S. Department of Energy

Lawrence

Livermore

National

Laboratory

\section{August 11, 2000}




\section{DISCLAIMER}

This document was prepared as an account of work sponsored by an agency of the United States Government. Neither the United States Government nor the University of California nor any of their employees, makes any warranty, express or implied, or assumes any legal liability or responsibility for the accuracy, completeness, or usefulness of any information, apparatus, product, or process disclosed, or represents that its use would not infringe privately owned rights. Reference herein to any specific commercial product, process, or service by trade name, trademark, manufacturer, or otherwise, does not necessarily constitute or imply its endorsement, recommendation, or favoring by the United States Government or the University of California. The views and opinions of authors expressed herein do not necessarily state or reflect those of the United States Government or the University of California, and shall not be used for advertising or product endorsement purposes.

This is a preprint of a paper intended for publication in a journal or proceedings. Since changes may be made before publication, this preprint is made available with the understanding that it will not be cited or reproduced without the permission of the author.

This report has been reproduced directly from the best available copy.

Available to DOE and DOE contractors from the

Office of Scientific and Technical Information

P.O. Box 62, Oak Ridge, TN 37831

Prices available from (423) 576-8401

http:/ / apollo.osti.gov/bridge/

Available to the public from the National Technical Information Service

U.S. Department of Commerce 5285 Port Royal Rd., Springfield, VA 22161 http://www.ntis.gov/

OR

Lawrence Livermore National Laboratory Technical Information Department's Digital Library http://www.llnl.gov/tid/Library.html 


\title{
Plasma Focusing of High Energy Density Electron and Positron Beams
}

\author{
J.S.T. $\mathrm{Ng}^{1}$, H. Baldis ${ }^{2}$, P. Bolton ${ }^{2}$, P. Chen ${ }^{1}$, D. Cline ${ }^{3}$, W. Craddock ${ }^{1}$, \\ C. Crawford ${ }^{4}$, F.J. Decker ${ }^{1}$, R.C. Field ${ }^{1}$, Y. Fukui ${ }^{3}$, V. Kumar ${ }^{3}$, R. Iverson ${ }^{1}$, \\ F. King ${ }^{1}$, R. Kirby ${ }^{1}$, T. Kotseroglou ${ }^{1}$, K. Nakajima ${ }^{6}$, R. Noble ${ }^{4}$, A. Ogata ${ }^{6}$, \\ P. Raimondi ${ }^{1}$, D. Walz ${ }^{1}$, A.W. Weidemann ${ }^{7}$ \\ ${ }^{1}$ Stanford Linear Accelerator Center, Stanford, California, 94809 \\ ${ }^{2}$ Lawrence Livermore National Laboratory, Livermore, California \\ ${ }^{3}$ University of California, Los Angeles, California, 90024 \\ ${ }^{4}$ Fermi National Accelerator Laboratory, Batavia, Mlinois \\ ${ }^{5}$ High Energy Accelerator Research Organization, Tsukuba, Ibaraki 905-0801 \\ ${ }^{6}$ Hiroshima University, Kagamiyama, Higashi-Hiroshima, 739-8526 Japan \\ ${ }^{7}$ University of Tennessee, Knoxville, Tennesee 97996
}

August 11, 2000

\begin{abstract}
We present results from the SLAC E-150 experiment on plasma focusing of high energy density electron and, for the first time, positron beams. We also present results on plasma lens-induced synchrotron radiation, longitudinal dynamice of plasma focusing, and laser- and beam-plasma interactions.

\section{INTRODUCTION}

The plasma lens was proposed as a final focusing mechanism to achieve high luminosity for future high energy linear colliders [1]. Previous experiments to test this concept were carried out with low energy density electron beams [2]. The goals of the E-150 experiment were to study plasma focusing for high energy, high density particle beams in the regime relevant to linear colliders, to obtain better understanding of beam-plasma interactions and bench-marking of computer codes for plasma lens designs, and to develop compact and economical plasma lens designs suitable for collider experiments. In this paper, after a description of the experimental setup, we present preliminary results obtained recently by the $\mathrm{E}$ 150 collaboration on plasma focusing of high energy density electron and positron beams. 
TABLE 1. FFTB electron and positron beam parameters for this experiment.

\begin{tabular}{ll|l}
\hline Parameter & Value & Units \\
\hline Bunch intensity & $1.5 \times 10^{10}$ & particles per pulse \\
Beam size & 5 to $8(X), 3$ to $5(\mathrm{Y})$ & $\mu \mathrm{m}$ \\
Bunch length & 0.7 & $\mathrm{~mm}$ \\
Beam energy & 29 & $\mathrm{GeV}$ \\
Normalized emittance & 3 to $5 \times 10^{-5}(\mathrm{X}), 0.3$ to $0.6 \times 10^{-5}(\mathrm{Y})$ & $\begin{array}{l}\mathrm{m} \mathrm{rad} \\
\mathrm{cm}^{-3}\end{array}$ \\
Beamn density & $\sim 7 \times 10^{16}$ & \\
\hline & & \\
& EXPERIMENTAL SETUP
\end{tabular}

The experiment was carried out at the SLAC Final Fodus Test Beam facility [3], The experiment operated parasitically with the PEP-II B-factory; the high energy electron and positron beams were delivered to the FFTB at $1-10 \mathrm{~Hz}$ from the SLAC linac. The beam parameters are summarized in Table 1 .

A layout of the beam line is shown in Figure 1. The beam size was measured using a wire-scanner system developed for previous SLC and the FFTB operations. A carbon fiber $4 \mu \mathrm{m}$ or $7 \mu \mathrm{m}$ in diameter was placed down stream of the plasma lens, at adjustable locations in a range of 3 to $30 \mathrm{~mm}$ from the center of the lens. The Bremsstrahlung photons emitted as the beam partiches scatter off the carbon fiber were detected in a Cherenkov type detector located $\sim 33 \mathrm{~m}$ down stream of the lens. The variation in photon yield as the beam "dithers" across the wire provides a measure of the transverse beam profile from which the beam size can be determined. Located behind the wire-scanner detector $\sim 35 \mathrm{~m}$ down stream of the lens, is a set of ionization chambers interleaved with polyethylene blocks used to monitor the synchrotron radiation emitted as a result of the strong bending of the beam particles by the plasma lens. This detector provides an independent measure of the focusing strength. Also, a Cherenkoy target is installed in the down stream electron beam line to allow streak camera diagnostics of the longitudinal plasma

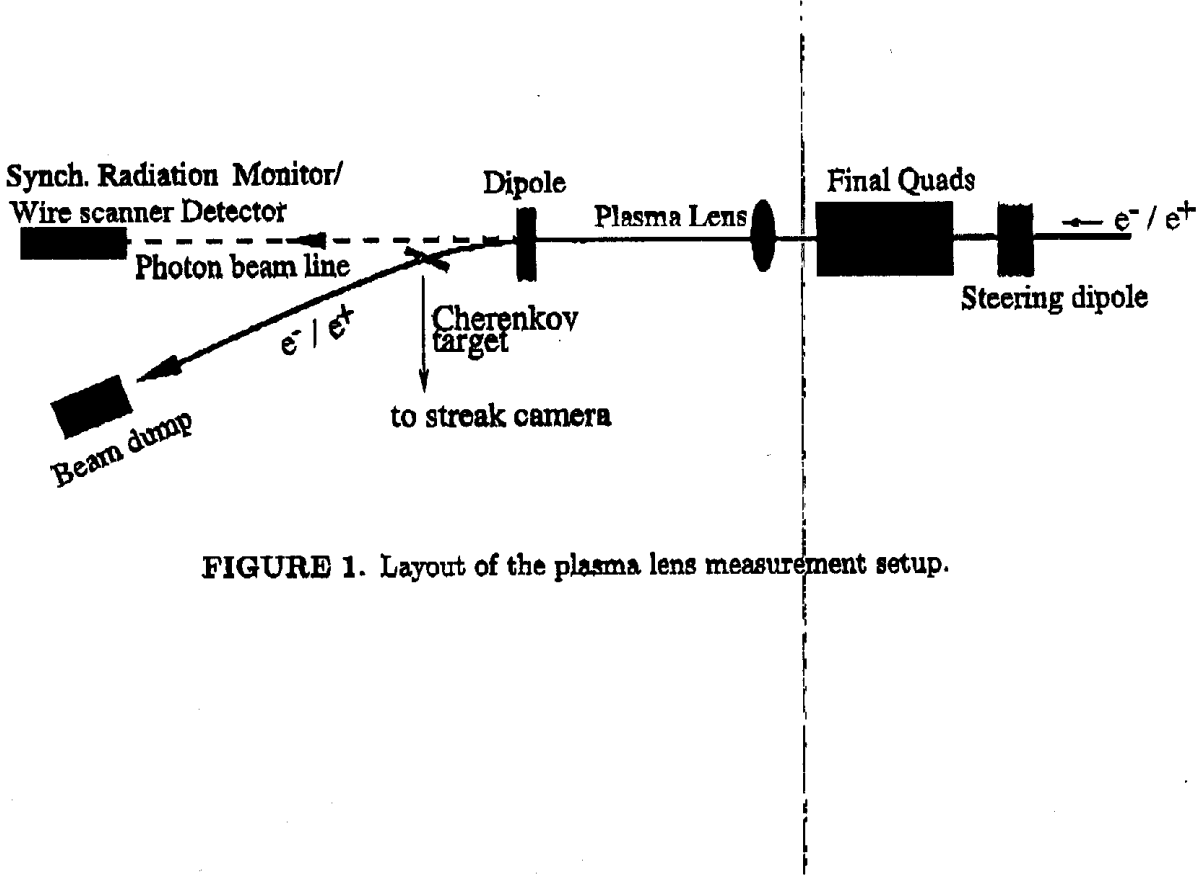




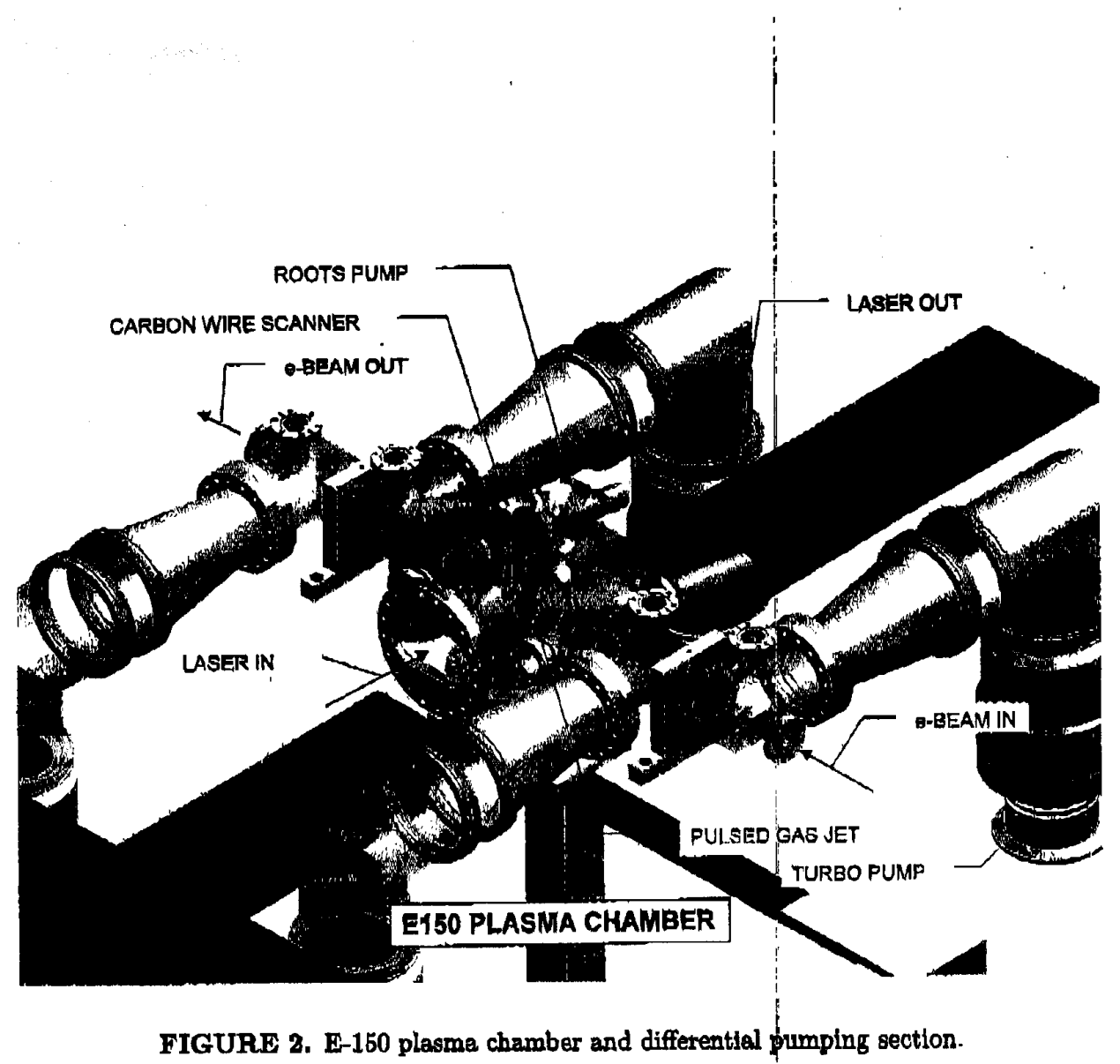

focusing dynamics.

To create the plasma lens, a short burst ( $800 \mu$ s duration) of neutral nitrogen or hydrogen gas injected into the plasma chamber by a fast-pulsing nozzle was ionized by a laser and/or the high energy beam. The solenoid valve ${ }^{1}$ operated at $0.5-2 \mathrm{~Hz}$. Its $0.8 \mathrm{~mm}$ orifice was matched to a parabolic-shaped nozzle $5 \mathrm{~mm}$ long with an opening of $3 \mathrm{~mm}$ diameter. The transverse profile of the gas-jet was obtained by scanning the high energy beam perpendicular to its axis. The gas-jet diameter which determines the plasma lens thicloness was found to be $3 \mathrm{~mm}$ at 1.4 and $3.4 \mathrm{~mm}$ away from the nozzle exit. The neutral density was determined by interferometry to be $4 \times 10^{18} \mathrm{~cm}^{-3}$ for $\mathrm{N}_{2}$ and $5 \times 10^{18} \mathrm{~cm}^{-3}$ for $\mathrm{H}_{2}$ at a plenum pressure of $1000 \mathrm{psi}$. The injected gas was evacuated by a differential pumping system which allows operation of the gas-jet while maintaining ultra-high vacuum in beam lines on either side of the chamber. A schematic drawing of the plasma. chamber is shown in Figure 2.

1) Model 91-222, Parker Hannifin Co. 


\section{RESULTS}

The results presented below were obtained from data taken during the Fall of 1999 with electron beams and the Spring of 2000 with positron beams. Measurements related to plasma focusing are presented first, followed by results on longitudinal focusing dynamics and laser- and beam-plasma interactions.

\section{Plasma focusing}

For a bunched relativistic beam traveling in vacuum, the Lorentz force induced by the collective electric and magnetic fields is nearly cancelled allowing it to propagate over kilometers without dispersing. An inltially quiescent plasma responds to the intruding charge and current in such a way that the plasma electron distribution is re-configured to neutralize the space charge of the beam and thereby cancel its radial electric field. For a positron beam, the plasma electrons are attracted into the beam volume thus neutralizing it; for an electron beam, the plasma electrons are expelled from the beam volume, leaving behind the less mobile positive ions which neutralize the beam. When the beam radius is much smaller than the plasma wavelength, the neutralization of the intruding beam current by the plasma return current is ineffective because of the small skin depth. This leaves the azimuthal magnetic field unbalanced which then "pinches" the beatm, resulting in focusing. In this experiment, typical plasma densities were of the order of $10^{18} \mathrm{~cm}^{-3}$, corresponding to a plasma wavelength of $\sim 30 \mu \mathrm{m}$ which is indeed much larger than the incoming beam radius.

\section{Beam self-ionization plasma fochusing}

As the high energy density beam intercepts the gas jet, a small fraction of the collisions between the beam particles and the neutral gas molecules resulted in ionization. The secondary electrons from this impact ionization process were accelerated by the intense collective field in the beam, trankverse to the direction of propagation, to further ionize the gas [4]. This beam self-ionization plasma was observed to focus the beam. That is, the head of the burich was able to ionize the gas while the core of the bunch was focused. The results for electron beams are shown in Flgure 3. The reduction in beam size was measured with wire scanning taken with the gas nozzle toggled off and on. The beam entvelope was also measured by adjusting the down stream position of the wire-scanner; a reduction of a factor two in beam spot area was observed.

The beam self-ionization efficiency was determined for nitrogen gas by comparing the plasma focusing effects in two data samples. One sample was taken with plenum pressure at $250 \mathrm{psi}$ with beam self-ionization and ionizdtion by a Terawatt laser system; while the other sample at 500 psi was taken with beam self-ionization only. Based on the laser performance and optical field ionization data [5], the Terawatt 


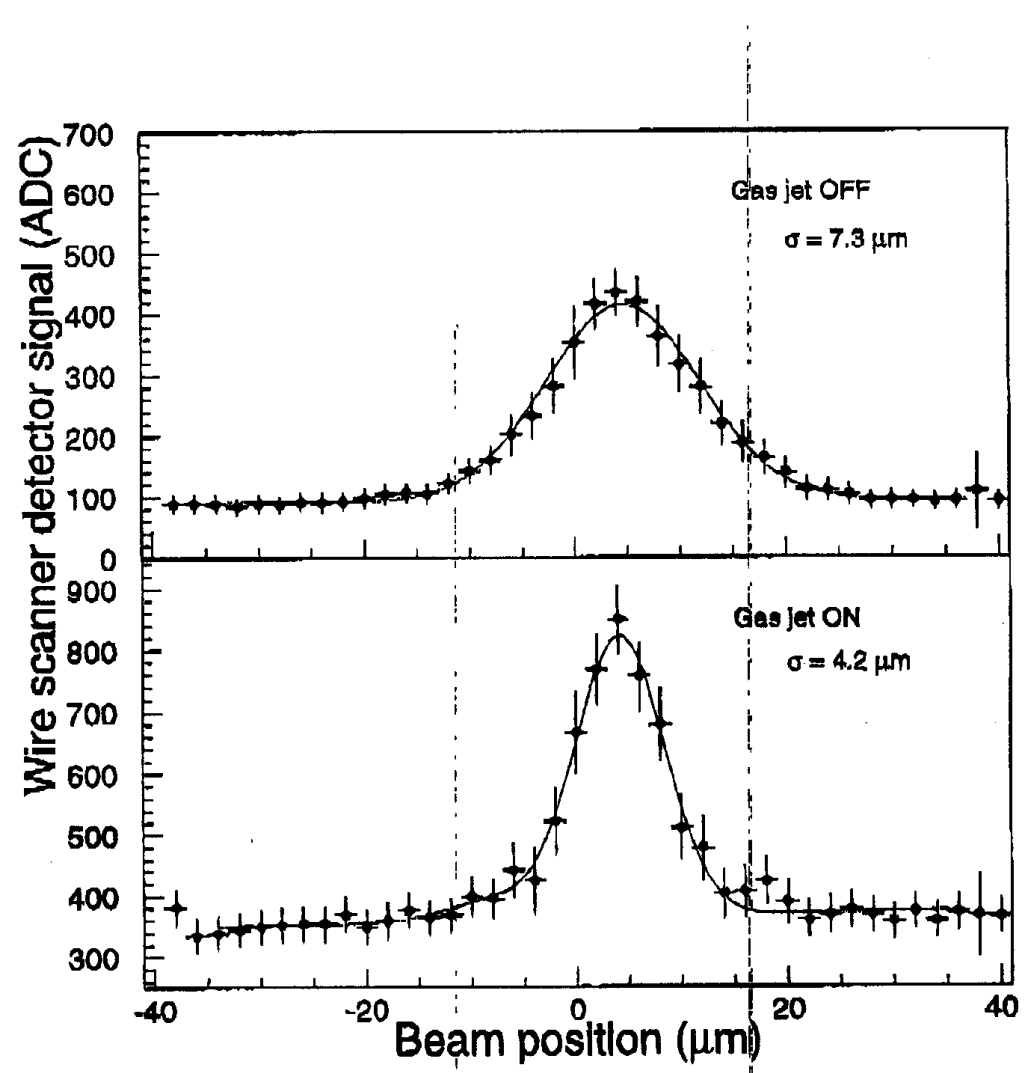

FIGURE 9. Wire scanner data showing focusing by a beam self-ionization plasma.

laser's ionization efficiency was estimated to be $10 \%$. The beam size reduction was observed to be the same for the two data samples at one location along the beam waist. Assuming the plasma densities to be the same in both data sets, we arrived at a beam ionization efficiency of $7 \%$, Note that this argument is only valid for low ionization yields.

\section{Laser avalanche ionization plasmalfocusing}

The results on laser pre-ionization plasma focusing presented here were obtained using a turn-key infrared laser system ${ }^{2}$. It delivered 1.5 Joules of energy per pulse of $10 \mathrm{~ns}$ FWHM while operating at $10 \mathrm{~Hz}$ with a wavelength of $1064 \mathrm{~nm}$. The laser light was brought to a line focus at the gas-jet; the plasma thus produced was approximately $0.5 \mathrm{~mm}$ thick as seen by the $e^{+} / e^{-}$beam.

The laser avalanche ionization process is similar to the beam self-ionization process. With the relatively long infrared laser pulse, the pulse front was able to ionize

2) QuantaRay GCR-3D10, Continuum Inc., CA. 
a fraction of the gas by multiple-photon absorption, while the resulting secondary electrons were accelerated, transverse to the laser's incident direction, to further ionize the gas resulting in on avalanche growth in plasma density. The ionization efficiency was determined by interfercmetry to be $45 \%$ in this case.

The results for laser (and beam) ionization plasma focusing of positron beams are shown in Figure 4. The measured beam spot size is shown as a function of the distance between the plasma lens and the wire scanner; the converging of the beam envelope towards a waist can be seen. The first plot shows the focusing for nitrogen gas at $800 \mathrm{psi}$, while the second shows the result for a hydrogen gas at 1100 psi plenum pressure. A reduction in beam spot size by a factor of two was observed with laser- and beam-induced ionization; while focusing was also observed with beam-induced ionization alone as in the case of electron beams discussed in the previous section.

\section{Synchrotron radiation induced by plasma focusing}

The synchrotron radiation induced by the strong bending of beam particles inside the plasma lens provides an independent measure of the plasma focusing strength. Since the plasma lens effect is transient, this radiation provides an "on-line" diagnostic of the focusing strength. The critical energy of the emitted synchrotron radiation scales with the strength of the focusing; a monitor was designed to measure the penetration profile, which depends on the energy, spectrum, of the photon flux.

During plasma focusing measurements, the photon flux detected in the synchrotron radiation monitor consisted of several components. There was radiation accompanying the beam, due to Bremsstrahlung of off-axis beam particles scattering off the beam pipe wall or upstream collimator jaws, and synchrotron radiation (with a critical energy $\sim 100 \mathrm{keV}$ ) emitted when the beam was bended towards the dump. This was measured with the gas-jet turned off and the wire scanner removed from the beam. The contribution from beam-gas Bremsstrahlung with the gas nozzle turned on was determined by first measuring the Bremsstrahlung penetration profile from the carbon wire alone, then scaling this profile to the signal measured in the last depth section of the monitor with the beam-associated background removed. After these background contributions were subtracted, an excess of photon signal beyond a depth of 3 radiation lengths was observed in the electron beam data, with nitrogen gas at $\mathbf{5 5 0}$ pisi plenum pressure, jand beam self-ionization. This excess was not observed when plasma focusing weak (from beam spot size measurements) as in the case of lowered plenum pressure (to below $100 \mathrm{psi}$, for example), or in the case of hydrogen (gas for which beang self-ionization was much less effective.

The penetration profile of the observed signal is consistent with that produced by synchrotron radiation with critical entergy of a few $\mathrm{MeV}$ according to Monte Carlo simulation. This value corresponds tọ a focusing gradient of the order of $10^{6} \mathrm{~T} / \mathrm{m}$, 
Plasma Focusing of Positron Beams
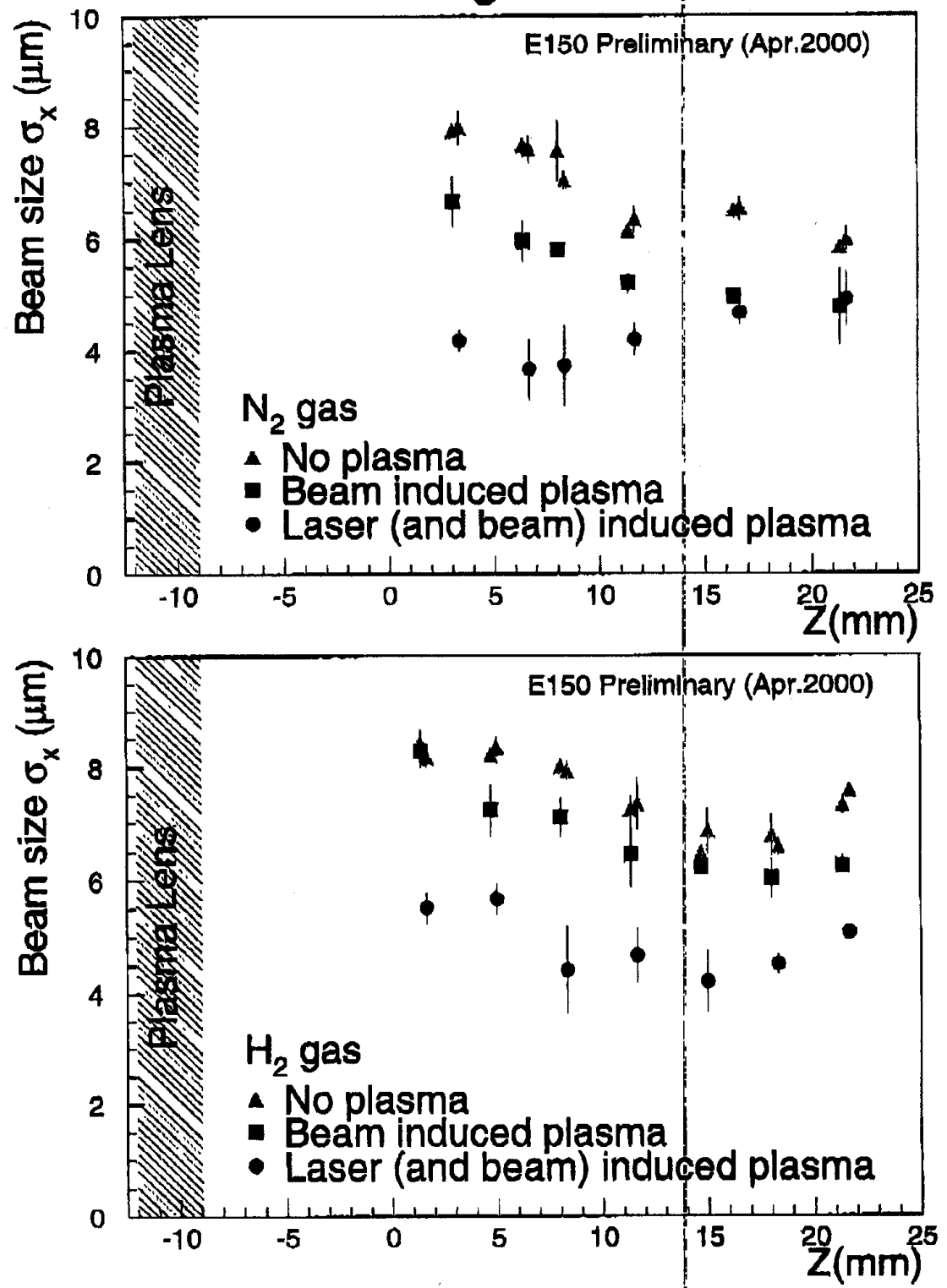

FIGUFE 4. The evolution of the beam envelope, with and without plasma focusing, is shown after the exit of the plasma lens for two types of gas. The axis of the gas-jet is at $\mathrm{Z}=-10.5 \mathrm{~mm}$. 
as expected for the plasma lens parameters in this experiment.

\section{Longitudinal focusing dynamics}

A streak camera with pico-second time resolution was used to probe the variation in plasma focusing along the bunch ${ }^{3}$. The Cherenkov light emitted as the beam passes through an gerogel target was imaged onto the streak camera. The vertical dimension of the beam was then measured in pico-second time slices along the bunch. The phase advance of the beam transport was that focusing at the plasma lens becomes defocusing at the aerogel target. Thus the increased beam divergence as a result of plasma focusing was measured, and therefore a stronger focusing at the plasma lens would result in a larger beam size at the Cherenkov target.

The results from data taken with positron beams are shown in Figure 5 , for nitrogen gas at two different pressures with beam self-iohization. The beam spot size in the vertical plane was measured with the gas-nbzzle turned on and off, and the difference is shown for time slices along the bunch. As expected, plasma focusing is significantly stronger at the longitudinal beam centroid. A detailed explanation of this result requires modeling of the beam self-ionlzation process which is currently under study.

\section{Laser- and beam-plasma interactions}

The laser avalanche ionization process has a finite build-up and decay time. By varying the laser pre-ionization timing before the beam arrival at the plasma, the state of the plasma can be probed using the high energylbeam. The signal in the synchrotron radiation monitor was measured as a function of this advanced timing. Since this signal was predominantly synchrotron radiation induced by plasma lens focusing, the measurement can also be used to study the interaction between the high energy beam and the plasma at various stages of formation and decay.

The result for positron beams and nitrogen gas at 1050 psi is shown in Figure 6. Time-zero corresponds to synchronization between the beam arrival time and the prompt-recombination time from signals detected in a photomultiplier. The synchrotron radiation monitor signal from beam self-ionization of the initial nitrogen gas was 300 ADC counts, while the beam-only background signal level was 80 ADC counts (as measured with the gas nozzle turned off).

A quantitative understanding of this delay curve requites detailed modeling of the laser- and beam-plasma interaction process. A qualitative explanation of this curve is given here. The initial rapid increase in synchrotron radiation monitor signal most likely corresponded to an increase in plasma, focúsing strength, peaking at

9) Courtesy of Mark Hogan of the SLAC E-157 experiment. 


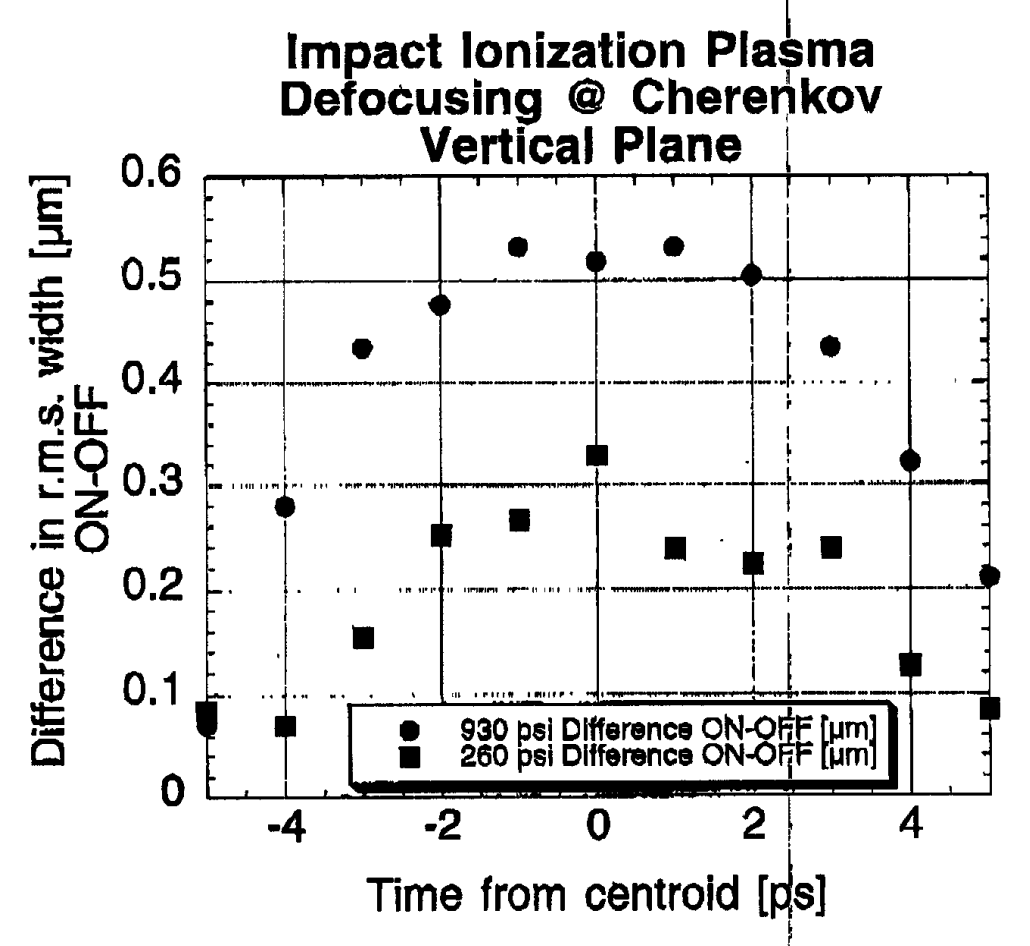

FIGURE 5. Streak camera diagnostics of plasma focusing longitudinal dyamics.

500 ns. This was followed by a sharp decrease to the background signal level, corresponding to an almost complete expulsion of the neutral and ionized gas volume by the sonic pressure of the laser "blast" wave. It should be noted that for each gas-jet pulse, a continuous stream of neutral gas molecules lasting more than $100 \mu s$ was injected into the vacuum chamber at sonic speed. So that at $1 \mu s$ the original laser iontzation plasma would have moved out of the beam interaction range. The rest of the curve shows a relaxation process. Initially, the gas rushed back to fill the void ( 1 to $9 \mu s$ range), giving rise to a locally increased gas pressure with a corresponding rise in beam-ionization plasma focusing signal. This was followed by a decay back to the initial quiescent level at a time delay of $15 \mu s$. Additional data has been collected since the Workshop, with varying pressures for nitrogen and hydrogen. A detailed simulation study should provide further insight into this plasma lens modulation phenomenon. 


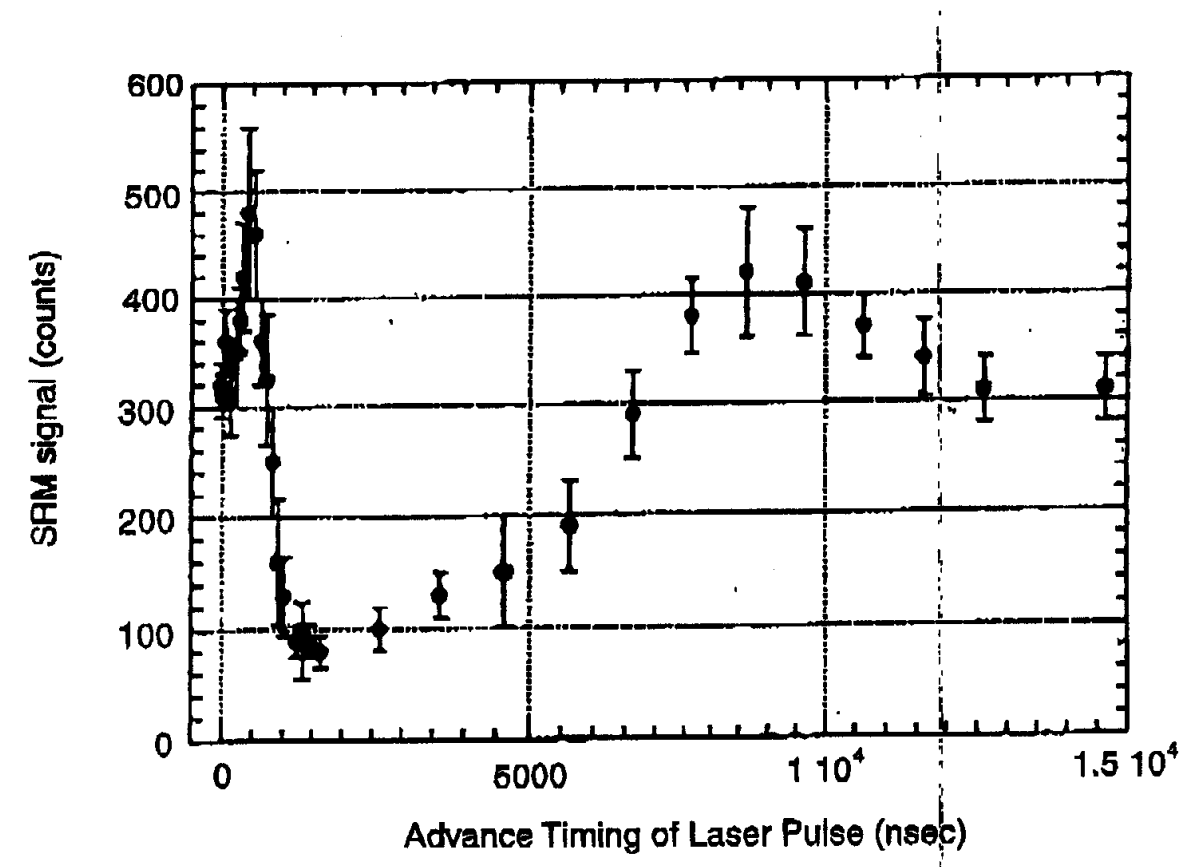

FIGURE 6. Synchrotron radiation monitor signal as a fundtion of the advanced laser pre-ionization timing with respect to beam arrival. No background subtraction was made.

\section{SUMMARY AND OUTLd,oK}

Results on plasma focusing of $29 \mathrm{GeV}$ electron and, for the flist time, positron beams are presented. Beam self-ionization turned out to be an economical method for producing a plasma lens; a factor of two reduction in the beam spot area was achieved with this method. The plasma focusing strength was also measured independently by monitoring the synchrotron radiation emitted by particles focused by the lens. The infrared laser with a 10-ns long pulse proved to be efficient in plasma production, resulting in the strong focusing of positron beams. The longitudinal focusing dynamics was diagnosed with a streak camera, and as expected the focusing was strongest at the longitudinal center of the bunch. The laser- and beam-plasma interaction was studied by varying the laser pre-ionization timing with respect to the beam axrival time; we observed that plasma focusing foas modulated as a result of this interaction.

Design studies for linear collider applications are just starting. The first issue to resolve is the effect of beam jitters on the achievable luminosity of plasma focused beams. Plasma lens parameters will need to be optimilzed also, which requires bench-marking of computer code and better understanding of the various plasma production processes. The experience gained in this experiment will serve as a basis for further engineering design studies for an eventual plasma lens application. 


\section{ACKNOWLEDGEMENT'}

This work was supported in part by the Department of Energy under contracts DE-AC02-76CH03000, DE-AC03-76SF00515, and W-7405-Eng-48; and by contributions from the US-Japan fund.

\section{REFERENCES}

1. P. Chen, Part. Accel., 20, 171(1987).

2. J.B. Rosenzweig et al, Phys. Fluids B 2, 1376(1990); H. Nakanishi et al., Phys. Rev. Lett, 66, 1870(1991); G. Hairapetian et al., Phys. Rev. Lețt. 72, 2403(1994); R. Govil et al., Phys. Rev. Lett. 83, 3202(1999).

3. V. Balakin et al., Phys. Rev. Lett. 74, 2479(1995).

4. R.J. Brigge and S. Yu, LLNL Report UCID-19399, May 1982 (unpublished).

5. B. Chang et al., Phys. Rev. A 47, No. 5, 4193(1993). 